OPEN ACCESS

Edited by:

Teresa A.P. Rocha-Santos, University of Aveiro, Portugal

Reviewed by:

Gerrit Renner,

Hochschule Niederrhein, Germany Monica F. Costa, Universidade Federal de Pernambuco,

Brazil

*Correspondence

Sherri A. Mason

mason@fredonia.edu

Specialty section: This article was submitted to Analytical Chemistry, a section of the journal

Frontiers in Chemistry

Received: 25 April 2018 Accepted: 20 August 2018 Published: 11 September 2018

Citation

Mason SA, Welch VG and Neratko J (2018) Synthetic Polymer Contamination in Bottled Water. Front. Chem. 6:407. doi: 10.3389/fchem.2018.00407

\section{Synthetic Polymer Contamination in Bottled Water}

\author{
Sherri A. Mason*, Victoria G. Welch and Joseph Neratko \\ Department of Chemistry, State University of New York at Fredonia, Fredonia, NY, United States
}

Eleven globally sourced brands of bottled water, purchased in 19 locations in nine different countries, were tested for microplastic contamination using Nile Red tagging. Of the 259 total bottles processed, 93\% showed some sign of microplastic contamination. After accounting for possible background (lab) contamination, an average of 10.4 microplastic particles $>100$ um in size per liter of bottled water processed were found. Fragments were the most common morphology (66\%) followed by fibers. Half of these particles were confirmed to be polymeric in nature using FTIR spectroscopy with polypropylene being the most common polymer type (54\%), which matches a common plastic used for the manufacture of bottle caps. A small fraction of particles (4\%) showed the presence of industrial lubricants. While spectroscopic analysis of particles smaller than 100 um was not possible, the adsorption of the Nile Red dye indicates that these particles are most probably plastic. Including these smaller particles (6.5-100 um), an average of 325 microplastic particles per liter of bottled water was found. Microplastic contamination range of 0 to over 10,000 microplastic particles per liter with $95 \%$ of particles being between 6.5 and 100 um in size. Data suggests the contamination is at least partially coming from the packaging and/or the bottling process itself. Given the prevalence of the consumption of bottled water across the globe, the results of this study support the need for further studies on the impacts of micro- and nano- plastics on human health.

Keywords: plastic pollution, microplastic, consumables, human health, FTIR, Nile Red, drinking water

\section{INTRODUCTION}

Plastic is defined as any synthetic or semi-synthetic polymer with thermo-plastic or thermoset properties, which may be synthesized from hydrocarbon or biomass raw materials (UNEP, 2016). Plastics production has seen an exponential growth since its entrance on the consumer stage, rising from a million tons in 1945 to over 300 million tons in 2014 (PlasticsEurope, 2015). Some of the features of plastic that make it so attractive from a manufacturing standpoint are of concern when it comes to its environmental impact. It is very light-weight allowing it to be easily transported over long distances, and it is durable being resistant to breakage and biodegradation. Its durability is inherently connected to its chemical structure. Being composed largely, if not entirely, of hydrocarbon chains, the lack of double bonds or other functional groups provides an inherent stability to its molecules, and its synthetic nature means that the vast majority of microorganisms haven't evolved to utilize plastic as a food source. Thus, while plastic will break into smaller and smaller particles via photo-oxidative mechanisms, the fundamental molecular structures of the material change very little throughout that process. Plastics become microplastics become nanoplastics, but they are all plastics, just of increasingly smaller size, allowing them to be more easily ingested and perhaps even cross the gastrointestinal tract to be transported throughout a living organism (Brennecke et al., 2015; Sharma and Chatterjee, 2017). 
With the rise in plastics manufacture, there has been an associated rise in plastic pollution of the external environment. The first reports date back to the early 1970's (Carpenter and Smith, 1972) and most famously within the world's oceans (e.g., Moore et al., 2001; Eriksen et al., 2014), but more recently plastic pollution has been found within freshwater lakes, inland seas, rivers, wetlands and organisms from plankton to whales (and nearly every species in between; Eriksen et al., 2013; Baldwin et al., 2016; Horton et al., 2017; Lusher et al., 2017).

As its ubiquity in the external environment has been increasing, this has lead more researchers to investigate various consumables for the presence of plastic. The first such study focused on bivalves intended for human consumption (Van Cauwenberghe and Janssen, 2014). More recent studies have focused on fish (such as anchovies), as well as mussels (Rochman et al., 2015; Tanaka and Takada, 2016; Lusher et al., 2017). Two studies have noted the presence of microplastics within beer (Liebezeit and Liebezeit, 2014; Kosuth et al., 2018). Starting with a 2015 study of Chinese Sea Salt brands, several additional studies have established the presence of microplastics within these human consumables as well (Yang et al., 2015; Iñiguez et al., 2017; Karami et al., 2017; Kosuth et al., 2018). The firstever investigation of plastic pollution within globally sourced tap water (a total 159 samples from seven geographical regions spanning five continents) was published just earlier this year (Kosuth et al., 2018).

As research into the occurrence of plastic pollution has increased, sampling and analysis methods are continually evolving as well. Within the aqueous environment, volumereduced (using neuston nets) or bulk sampling followed by density separation, filtration/sieving and visual identification have been the most commonly employed methods (Hidalgo-Ruz et al., 2012). Given the time-consuming nature of these methods of sample processing, as well as the potential for misidentification using visual cues alone, one focus area for plastics pollution research (especially at the micro- and nano- scale) is development of methods for high-throughput with increased polymeric confirmation. Several recent studies have supported the use of Nile Red (NR) as an accurate stain for the rapid detection and quantification of microplastics given its selectivity adsorption and fluorescent properties. Maes et al. (2017) specifically tested the preferential adsorption of NR for polymeric materials relative to common organic (algae, seaweeds, wood and feathers) and inorganic (shells) environmental contaminants. Like Maes et al. (2017) and Erni-Cassola et al. (2017) validated the use of this stain with analysis using FTIR to verify the polymeric content of fluorescing particles. Both of these studies concluded from their efforts that NR can be used for the rapid detection of microplastics without the need for additional spectroscopic analysis (thereby reducing the time needed to analyze an environmental sample). These studies suggest that the adsorption of NR alone is sufficient to identify a particle as polymeric in nature. A conclusion further supported by the inclusion of this method within the recent review of analytical methodologies for microplastic monitoring by Renner et al. (2018).

Here we present a study utilizing Nile Red for the detection of microplastic within 11 globally- sourced brands of bottled water. In total 259 bottles of water from 11 brands were processed across 27 different lots (an identification number assigned by a manufacturer to a particular production unit) purchased from 19 locations in nine countries. For 10 brands we tested 2-3 lots each, while for one brand only one lot was tested. Within each lot, we generally tested 10 bottles (bottle volume $500-600 \mathrm{~mL}$ each) from the case. However, for one lot, several bottles from the case were seized by customs allowing only nine bottles to be tested, while for two other lots the volume of water per bottle was significantly greater (0.750-2 L) and thus only four (2 L bottles) or six bottles ( $750 \mathrm{~mL}$ bottles) were processed. One of the bottled water lots was packaged in glass (Gerolsteiner, $750 \mathrm{~mL}$, six glass bottles processed); all other samples were packaged in plastic. All bottles had plastic bottle caps.

\section{MATERIALS AND METHODS Sample Collection}

Sample lots were procured with an eye to geographic diversity (five continents are represented), size of the national packaged drinking water market (China, USA, Brazil, India, Indonesia, Mexico), and high per captia consumption of packaged drinking water (Lebanon, Mexico, Thailand, USA; Table 1). Leading international brands in this study included Aquafina, Dasani, Evian, Nestle Pure Life, and San Pellegrino. Leading national brands included Aqua (Indonesia), Bisleri (India), Epura (Mexico), Gerolsteiner (Germany), Minalba (Brazil), and Wahaha (China).

As many bottled water brands are simply filtered municipal tap water, sample lots were purchased from a number of locations to increase the likelihood of diverse bottling sources. For example, cases of the Mexican brand Epura were purchased from Tijuana in Baja California state, Reynosa on the Texas border (1,200 miles east of Tijuana), and Mexico City (1,400 miles south of Tijuana). This pattern is repeated with the other brands.

\section{Sample Processing}

The bottles within most (9 out of 11 brands) lots came in containers of $500-600 \mathrm{~mL}$ per bottle, while two of the brands contained $0.75-2 \mathrm{~L}$ per bottle. For those samples with 500-600 mL per bottle, 10 bottles were randomly chosen from the lot, while for the $750 \mathrm{~mL}$ samples, six bottles were chosen, and for the $2 \mathrm{~L}$ sample, four bottles were randomly chosen, and placed under a laminar flow fume hood. While under the fume hood, each bottle was opened and injected with a specific volume of Nile Red solution (prepared in acetone to $1 \mathrm{mg} \mathrm{mL}-1$ ) to yield a working concentration of $10 \mathrm{ug} \mathrm{mL-1}$ (Maes et al., 2017) and re-capped. Nile Red adsorbs to the surface of plastics, but not most naturally occurring materials, and fluoresces under specific wavelengths of light (Erni-Cassola et al., 2017). Bottles were allowed to incubate with the injected dye for at least $30 \mathrm{~min}$. The bottled water was then vacuum filtered through a glass fiber filter (Whatman grade 934-AH, $55 \mathrm{~mm}$ diameter, $1.5 \mathrm{um}$ pore).

Filters were examined under an optical microscope (Leica EZ4HD, 8-40× zoom, integrated 3 Mpixel camera) using a blue crime light (Crime-Lite 2, 445-510 nm, Foster \& Freeman) to elicit fluorescence, which was visualized through orange filter 
TABLE 1 | Selected market assessment data utilized to determine the countries of origin and brands tested within this study.

\begin{tabular}{|c|c|c|c|c|c|}
\hline \multirow[b]{2}{*}{ Brand } & \multirow[b]{2}{*}{ Parent company } & \multirow[b]{2}{*}{ Country } & \multicolumn{2}{|c|}{ Brand sales ranking } & \multirow[t]{2}{*}{ Country sales ranking in world } \\
\hline & & & In country & In world & \\
\hline Aqua & Danone (France) & Indonesia & 1 & 3 & 4 (by volume) \\
\hline Aquafina & Pepsico & USA & 2 & 7 & 2 (by volume) \\
\hline Bisleri & Bisleri (Indian) & India & 1 & 10 & 6 (in sales) \\
\hline Dasani & Coca-Cola & USA & 1 & 4 & 2 (by volume) \\
\hline Epura & Proprietary brand of GEPP & Mexico & 1 & - & 1 (per capita) \\
\hline Evian & Danone & $\begin{array}{l}\text { USA } \\
\text { United Kingdom } \\
\text { France }\end{array}$ & $\begin{array}{l}1 \text { (UK) } \\
2 \text { (France) }\end{array}$ & 3 & 1 (in sales) \\
\hline Gerolsteiner & $\mathrm{GmbH} \& \mathrm{Co} . \mathrm{KG}$ & GERMANY & 1 & - & $\begin{array}{l}4 \text { (per capita) } \\
8 \text { (in sales) }\end{array}$ \\
\hline Minalba & Edson Queiroz Group & Brazil & - & - & 5 (in sales) \\
\hline Nestle Pure Life & Nestle & Lebanon & 1 & 1 (parent company) & - \\
\hline San Pellegrino & Nestle & Italy & - & 1 (parent company) & $\begin{array}{l}3 \text { (per capita) } \\
9 \text { (in sales) }\end{array}$ \\
\hline Wahaha & Hangzhou Wahaha Group & China & 1 & 1 & 1 (by volume) \\
\hline
\end{tabular}

Dashes (-) indicate missing information.

viewing googles (Foster \& Freeman, $529 \mathrm{~nm}$ ). All particles larger than $\sim 100$ um (which are large enough to be visible to the naked eye and manipulated with tweezers) were photographed, enumerated and typed with respect to morphology (Fragment, Fiber, Pellet, Film, or Foam). Additionally the first 3-5 particles were analyzed via FTIR (PerkinElmer Spectrum Two ATR; $450 \mathrm{~cm}-1$ to $4,000 \mathrm{~cm}-1,64$ scans, $4 \mathrm{~cm}-1$ resolution; ATR correction) to confirm polymeric identity (Spectrum 10 software suite).

After removal of all particles $>100 \mathrm{um}$, the filter with fluorescing particles was photographed $(8 \times$ zoom $)$ through an orange camera filter (Foster \& Freeman, $62 \mathrm{~mm}$ diameter, $529 \mathrm{~nm}$ ) in four separate quadrants. To ensure no overlap of the quadrant photographs identification marks were made on the filters prior to turning the filter 90 degrees to take the subsequent photo. In fact, given the zoom factor of the microscope, quadrant photos did not obtain full (100\%) coverage of the filter. Each photographed quadrant was analyzed using a software program entitled "Galaxy Count" developed by a former astrophysicist for this specific purpose and briefly described here. Given the fluorescing particles relative to the non-fluorescing background, "Galaxy Count" is able to enumerate the number of particles (as bright spots) in order to quantifying the number of smaller microplastics. To do this, the operator of the software sets a threshold value which is used to convert the quadrant images to black (background filter) and white (fluorescing particles). The software then digitally counts the number of white spots ("stars") against the dark background ("the night sky"). At the $8 \times$ magnification in which the quadrant photos were taken, 1 pixel was equal to $6.5 \mathrm{um}$. Thus, while the filter pore size was $1.5 \mathrm{um}$, the smallest size particle visualized through the use of the combination of photography and software was $6.5 \mathrm{um}$. There could certainly be particles smaller than $6.5 \mathrm{um}$, but the method employed here would not be able to assess their presence. Due to the programmatic setting of the threshold value, all digital counts were conducted by two different researchers working independently of one another to account for possible variability.
Microplastic counts for particles $>100$ um (referred to as "NR + FTIR confirmed particles") are reported for each bottle. These particles are the ones that were further analyzed by FTIR and thus the types of polymers are also reported. Smaller microplastic particles (6.5-100 um; referred to as "NR tagged particles"), counted using the "Galaxy Count" software, are similarly reported for each bottle by summing over the four quadrants (each quadrant being reported as the average of the two researchers).

\section{Quality Assurance and Quality Control}

As the "Galaxy Count" software was created specifically for this project in order to verify its accuracy four solutions were created using DI water containing $0,20,50$ or 100 polyethylene microspheres (Cospheric, PE micropheres, $D=1.25 \mathrm{~g} \mathrm{~mL}-1$, 75-90 um diameter). These solutions were created by one researcher, but processed "blind" by another researcher in a manner identical to the samples themselves (NR injection, incubation, filtering, quadrant photographing and analysis by the "Galaxy Count" software). Additionally the analysis of all filter quadrants by the "Galaxy Count" software for all samples were conducted "blindly" by two separate researchers. These two counts were compared to one another for accuracy, in addition to being averaged for reported numbers.

In order to prevent/reduce potential contamination throughout the sample processing from external sources, such as airborne fibers, work occurred in a laminar airflow cabinet (Mott manufacturing, Phoenix Controls, serviced annually in September) and the workspace was wiped down every week. All glassware was covered with a watch glass when not in use and washed thoroughly between trials. Filters were inspected under a microscope prior to use, and a cotton lab coat and sterling nitrile powder free exam gloves were worn throughout the experimental procedure.

To account for possible lab contamination that could be coming from atmospheric deposition, the chemicals used, the 
glassware or other aspects of the testing environment, lab blanks containing deionized water (used to wash all glassware) or acetone (used to prepare the Nile Red solution) were processed in a manner identical to the samples themselves. Particle densities within samples were reduced based upon the average densities across all lab blanks.

\section{RESULTS \\ Overview}

A total of 259 individual bottles from across 11 different brands and 27 different lots were analyzed for microplastic particulate, subdivided into two size fractions: so-called "NR + FTIR confirmed particles," which are $>100$ um, and "NR tagged particles," which are 6.5-100 um. As quadrant photos did not provide full (100\%) coverage of the filter, it is likely that "NR tagged particles" are underestimated. Since individual bottles contained varied water volumes, from $500 \mathrm{~mL}$ to $2 \mathrm{~L}$, absolute counts for each bottle and size fraction were divided by sample volume to calculate (raw) densities of microplastic per liter (microplastic particles/L or MPP/L).

Thirteen lab blanks using laboratory deionized water or acetone were processed using methods identical to those for the bottled water samples. For "NR + FTIR particles" ( $>100 \mathrm{um}$ ) the average density was found to be $4.15 \mathrm{MPP} / \mathrm{L}$, with a range of 0-14 MPP/L, while within the smaller "NR tagged particles" (6.5-100 um) the average density was $23.5 \mathrm{MPP} / \mathrm{L}$, with a range of 7-47 MPP/L. Reported microplastic densities for the bottled water samples are calculated (by size fraction) from raw densities less the average from laboratory blanks (Table 1). If raw densities had less than or equal quantities relative to the laboratory blanks, their values were set to zero. Given that quadrant photos did not obtain full $(100 \%)$ coverage of the filter and that raw densities were reduced by lab blanks, reported densities are expected to be reasonable but conservative accounting of microplastic contamination. Total densities were calculated by summing across the size fractions (Table 2).

Seventeen bottles out of the 259 bottles analyzed $(\sim 7 \%)$ showed no microplastic contamination in excess of possible laboratory influence indicating that $93 \%$ of the bottled water tested showed some sign of microplastic contamination. The densities of microplastic contamination are quite variable ranging from the 17 bottles with no contamination to one bottle that showed an excess of 10,000 microplastic particles per liter (Table 2). The variabilities seen in the individual bottles, even among the same lot and brand, is similar to what is seen in sampling open bodies of water (Yonkos et al., 2014). Patterns in such sampling can be rather stochastic due to the large number of factors that can affect the occurrence of plastic particles (especially at the microscale), like particle-fluid dynamics, as well as variabilities within the manufacturing process itself, leading to the large variabilities seen within the samples. This erraticism highlights the need for large sample sizes, such as that employed here, in order to average across the variabilities to produce a realistic depiction.

Table 3 provides the mean (by size fraction and total), as well as the minimum and maximum, microplastic densities (in MPP/L) for each lot averaged across all the bottles tested.
When averaging across the individual bottles, all 27 lots tested showed some quantity of microplastic contamination (Table 2). Within brands there is significant variability between different lots, which could be owing to a number of factors, such as water source, different bottling facilities, or the conditions and/or length of time involved in shipping from bottling facilities to purchase location. The 17 individual bottles that showed no microplastic contamination in excess of possible laboratory background (Table 1) originated from seven lots ( 25\%) of the 27 tested. Thus, microplastic contamination was found within all bottles in $75 \%$ of the lots analyzed.

When averaged across all lots and all brands, $325 \mathrm{MPP} / \mathrm{L}$ were found within the bottled water tested [broken down as an average of $10.4 \mathrm{MPP} / \mathrm{L}$ occurring within the larger size range (>100 um) and an average $315 \mathrm{MPP} / \mathrm{L}$ within the smaller size range $(6.5-100 \mathrm{um})]$. While all bottled water lots tested showed some sign of microplastic contamination (Table 2), there was significant variation among the brands (Figure 1). Averaging across lots by brand, Nestle Pure Life and Gerolsteiner showed the highest average densities at 930 and $807 \mathrm{MPP} / \mathrm{L}$, respectively, while San Pellegrino and Minalba showed the lowest microplastic contamination with 30.0 and $63.1 \mathrm{MPP} / \mathrm{L}$, respectively (Figure 1). Error bars in Figure 1 represent one standard deviation and are quite large given the large variability among the individual bottles for each lot (Table 2), as well as the variation among lots of the same brand (Table 3 ).

Of all the lots tested, only one was packaged in glass rather than plastic: Gerolsteiner (NV No. AC-51-07269). While these samples revealed microplastic contamination, they did so at lower level as compared to the other lots (Tables 2, 3). Further, the same brand of water but packaged in plastic instead of glass was also tested (Gerolsteiner, 07.142018 2). While both of these packaged waters have the same water source, there was considerably less microplastic contamination within the water bottled in glass as compared to that packaged in plastic (204 vs. 1,410 MPP/L, respectively). This indicates that some of the microplastic contamination is likely coming from the water source, but a larger contribution might be originating from the packaging itself.

\section{NR + FTIR Confirmed Particles (>100 um)}

In total nearly 2,000 microplastic particles $>100$ um were extracted from all of the filters, with nearly 1,000 ( 50\%) being further analyzed by FTIR. Obtained FTIR spectra (after applied ATR correction) were compared to libraries of known spectra using the included PerkinElmer Spectrum 10 software suite in order to confirm and identify the polymeric content of the particles. All particles analyzed were either best matched to a polymer, plastic additive or known plastic binder providing additional supporting evidence that Nile Red selectively adsorbed to microplastic particles within the bottled water. With this spectroscopic confirmation, it can be concluded that on average each bottle of water contains at least 10.4 MPP/L (Table 3). While this analysis confirmed the polymeric nature of these particles, a match of $70 \%$ or greater was required in order to assign polymer identity. In total over 400 particles (20\% of all extracted plastic particles $>100 \mathrm{um}$ and $40 \%$ of those analyzed by FTIR) met this 


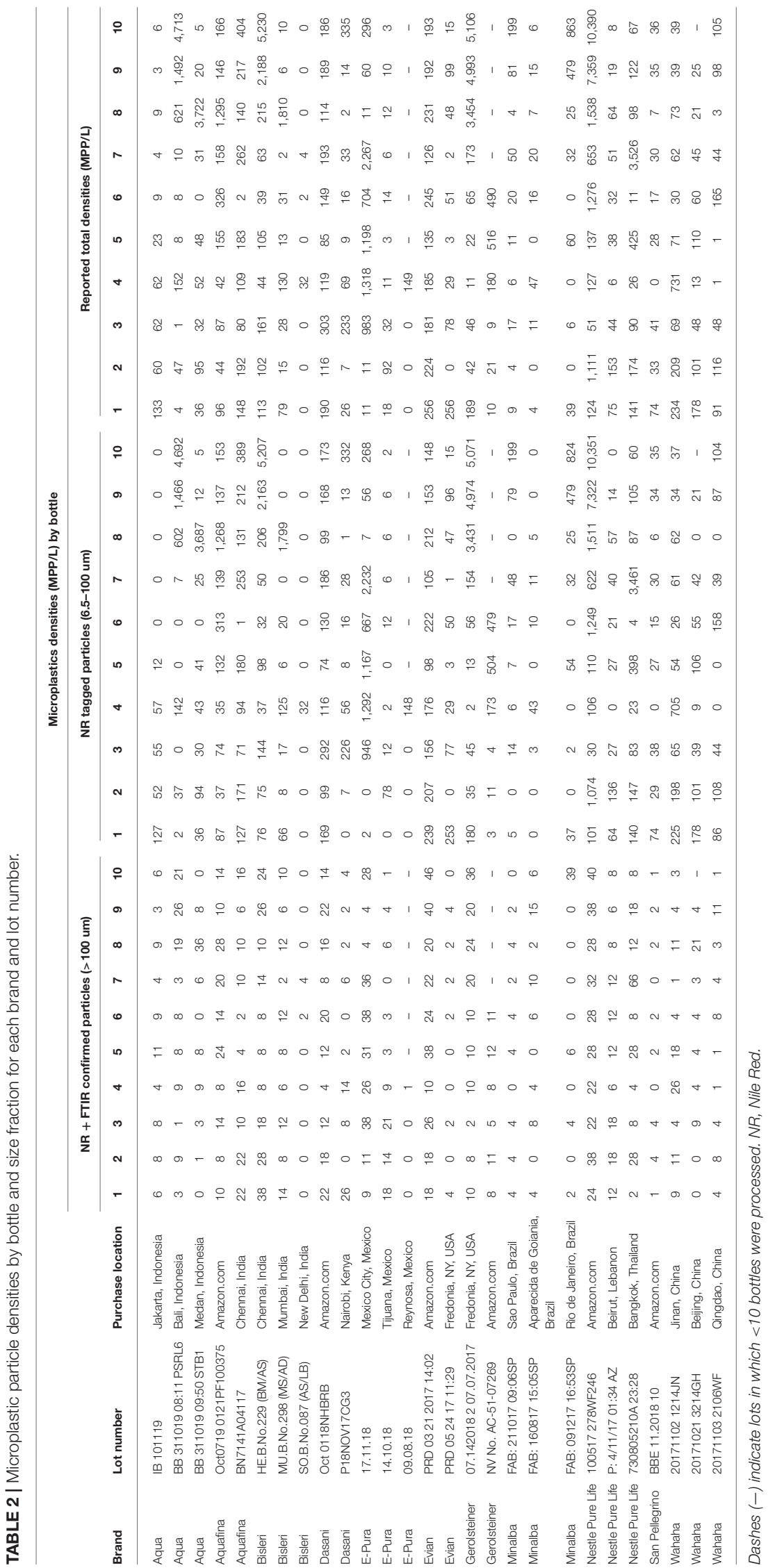


TABLE 3 | Microplastic densities (MPP/L), by size fractions and total, averaged across all bottles within the same lot.

\begin{tabular}{|c|c|c|c|c|c|c|c|}
\hline \multirow[b]{3}{*}{ Brand } & \multirow[b]{3}{*}{ Lot } & \multirow[b]{3}{*}{ Purchase location } & \multicolumn{5}{|c|}{ Average microplastic densities (MPP/L) } \\
\hline & & & $\begin{array}{c}\text { NR + FTIR confirmed } \\
\text { particles }\end{array}$ & NR tagged particles & Total & & \\
\hline & & & (>100 um) & (6.5-100 um) & Average & Minimum & Maximum \\
\hline Aqua & IB 101119 & Jakarta, Indonesia & 6.68 & 30.4 & 37.1 & 3 & 133 \\
\hline Aqua & BB 311019 08:11 PSRL6 & Bali, Indonesia & 10.5 & 695 & 705 & 1 & 4,713 \\
\hline Aqua & BB 311019 09:50 STB1 & Medan, Indonesia & 6.93 & 397 & 404 & 0 & 3,722 \\
\hline Aquafina & Oct0719 0121PF100375 & Amazon.com & 14.8 & 237 & 252 & 42 & 1,295 \\
\hline Aquafina & BN7141A04117 & Chennai, India & 11.6 & 162 & 174 & 2 & 404 \\
\hline Bisleri & HE.B.No.229 (BM/AS) & Chennai, India & 18.0 & 808 & 826 & 39 & 5,230 \\
\hline Bisleri & MU.B.No.298 (MS/AD) & Mumbai, India & 8.85 & 204 & 213 & 2 & 1,810 \\
\hline Bisleri & SO.B.No.087 (AS/LB) & New Delhi, India & 0.57 & 3.15 & 3.72 & 0 & 32 \\
\hline Dasani & Oct 0118NHBRB & Amazon.com & 14.6 & 150 & 165 & 85 & 303 \\
\hline Dasani & P18NOV17CG3 & Nairobi, Kenya & 6.28 & 68.3 & 74.6 & 2 & 335 \\
\hline E-Pura & 17.11 .18 & Mexico City, Mexico & 22.3 & 664 & 686 & 11 & 2,267 \\
\hline E-Pura & 14.10 .18 & Tijuana, Mexico & 7.76 & 12.2 & 20.0 & 3 & 92 \\
\hline E-Pura & 09.08.18 & Reynosa, Mexico & 0.21 & 37.1 & 37.3 & 0 & 149 \\
\hline Evian & PRD 03212017 14:02 & Amazon.com & 26.0 & 171 & 197 & 126 & 256 \\
\hline Evian & PRD $05241711: 29$ & Fredonia, NY, USA & 1.51 & 56.7 & 58.2 & 0 & 256 \\
\hline Gerolsteiner & 07.142018207 .07 .2017 & Fredonia, NY, USA & 14.8 & 1,396 & 1,410 & 11 & 5,106 \\
\hline Gerolsteiner & NV No. AC-51-07269 & Amazon.com & 8.96 & 195 & 204 & 9 & 516 \\
\hline Minalba & FAB: 211017 09:06SP & Sao Paulo, Brazil & 2.56 & 37.5 & 40.1 & 4 & 199 \\
\hline Minalba & FAB: 160817 15:05SP & Aparecida de Goiania, Brazil & 5.30 & 7.19 & 12.5 & 0 & 47 \\
\hline Minalba & FAB: 091217 16:53SP & Rio de Janeiro, Brazil & 5.01 & 145 & 150 & 0 & 863 \\
\hline Nestle Pure Life & 100517 278WF246 & Amazon.com & 29.8 & 2,247 & 2,277 & 51 & 10,390 \\
\hline Nestle Pure Life & $P: 4 / 11 / 17$ 01:34 AZ & Beirut, Lebanon & 11.0 & 38.2 & 49.3 & 6 & 153 \\
\hline Nestle Pure Life & 730805210A 23:28 & Bangkok, Thailand & 18.0 & 450 & 468 & 11 & 3,526 \\
\hline San Pellegrino & BBE 11.201810 & Amazon.com & 1.68 & 28.6 & 30.3 & 0 & 74 \\
\hline Wahaha & 20171102 1214JN & Jinan, China & 9.10 & 147 & 156 & 30 & 731 \\
\hline Wahaha & $201710213214 \mathrm{GH}$ & Beijing, China & 5.53 & 61.2 & 66.7 & 13 & 178 \\
\hline Wahaha & $201711032106 W F$ & Qingdao, China & 4.40 & 62.7 & 67.1 & 1 & 165 \\
\hline
\end{tabular}

Minimum and maximum densities within the lot are also provided. NR, Nile Red.

threshold for identity confirmation and only those results are presented here

Polypropylene was found to be the most common polymeric material (54\%) with Nylon being the second most abundant (16\%; Figure 2). Polypropylene is a polymer often used to make plastic bottle caps, along with polyethylene, which corresponded to $10 \%$ of the particles analyzed. Interestingly, $4 \%$ of retrieved particles were found to have signatures of industrial lubricants coating the polymer (not shown).

As is common practice in plastic pollution research, all microplastics $>100$ um were visually characterized according to their morphology: Fragment, Fiber, Pellet, Film, or Foam. Fragments were found to be the most common type of particle (66\%), followed by fibers (13\%) and films (12\%; Figure 3). The 13\% of particles described as fibers (Figure 3) compares well with the $17 \%$ of particles that were confirmed by FTIR to be composed of fiberous polymers, most notably Nylon (Figure 2).

\section{NR Tagged Particles (6.5-100 um)}

In order to verify the effectiveness of the "Galaxy Count" software to count microplastics smaller than $\sim 100 \mathrm{um}$, the software was tested using solutions with known quantities $(0,20,50$ or 100) of microspheres (diameters 75-90 um) processed in a manner identical to all samples and lab blanks. The "Galaxy Count" of fluorescing particles on the filter quadrant photos agreed very well with the actual count of particles included within the solutions (Figure 4). The excellent agreement with these test solutions supports the use of this tool for quantifying the numbers of smaller particles within the bottled waters analyzed, while the y-intercept of the least-squares fit further supports that the study is likely undercounting particles, especially within this smallest size range.

All counts using the "Galaxy Count" software were conducted independently by two different researchers owing to possible variabilities in software settings. As shown in Figure 5, the agreement in counts between the two researchers is excellent providing additional support to the effectiveness and validity 


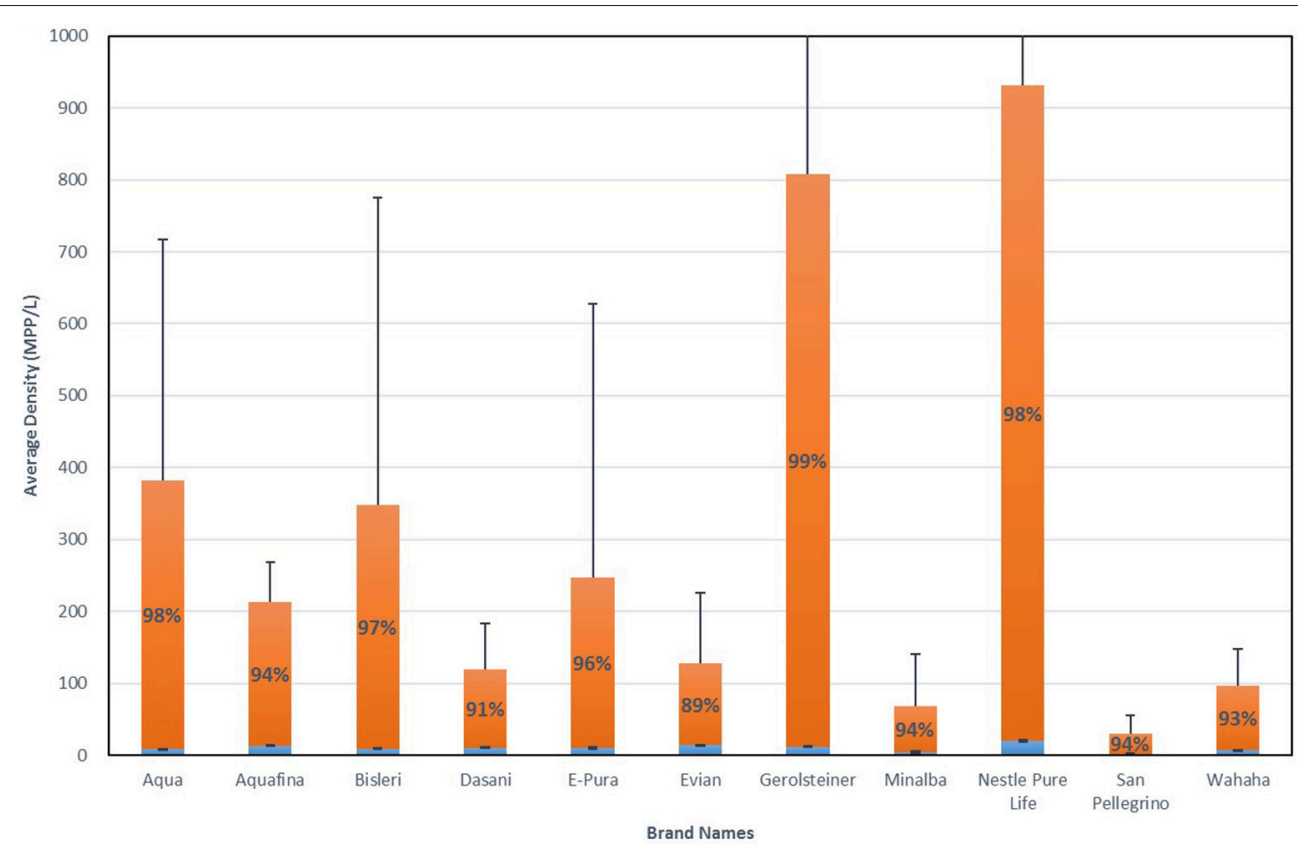

FIGURE 1 | Microplastic density averaged across individual bottles and lots by brand. Blue bars are densities for "NR + FTIR confirmed particles" (>100 um); Orange bars are for "NR tagged particles" (6.5-100 um). Error bars are one standard deviation. Percentages are for the contribution to the total for "NR tagged particles" (6.5-100 um); Contribution of larger particles can be inferred.

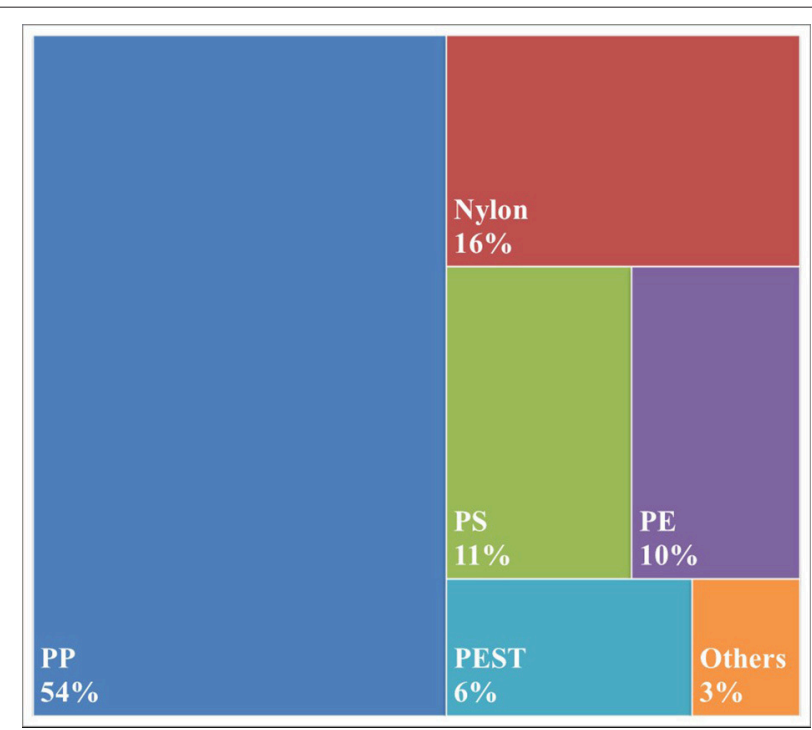

FIGURE 2 | Polymeric content of microplastic particles >100 um found within bottled water. PP, polypropylene; PS, polystyrene; PE, polyethylene; PEST, polyester + polyethylene terephthalate; Others includes Azlon, polyacrylates and copolymers.

in using the software to count the smaller particles within the bottled water.

Given the limitations of the lab, particles $<100$ um (the socalled "NR tagged particles") were not able to be confirmed as polymeric through spectroscopic analyses (FTIR and/or Raman

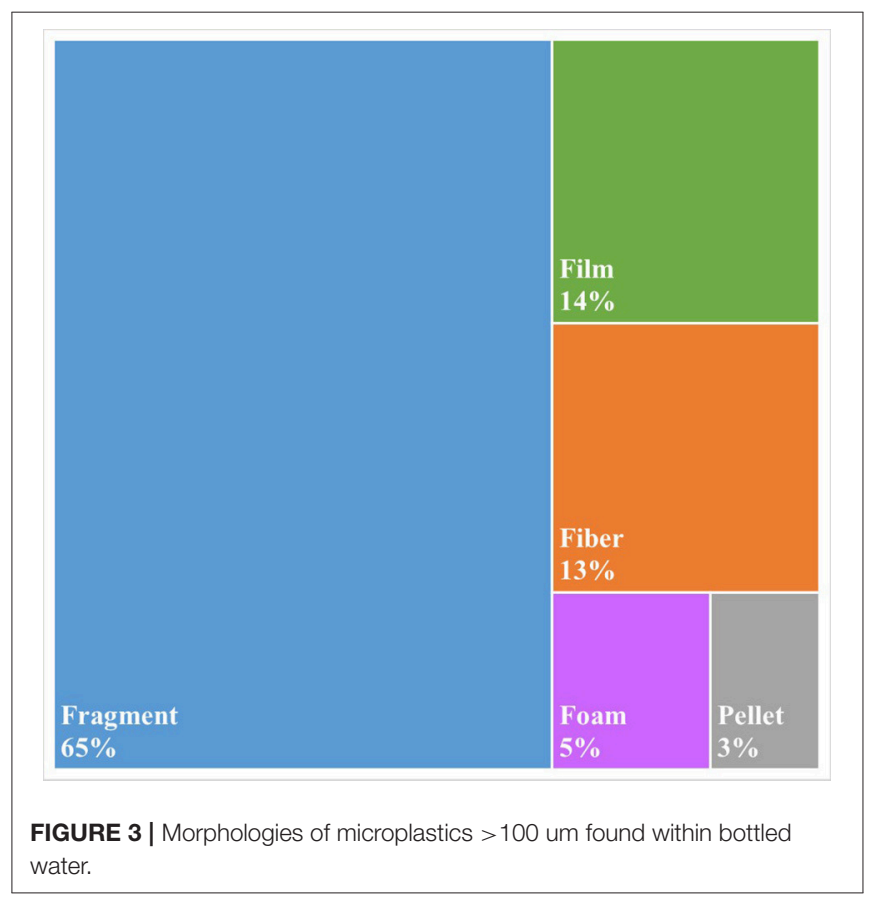

spectroscopy). However, in testing of various stains and dyes that could be employed for microplastic detection and analysis within environmental samples with a greater potential for misidentification and false positives (i.e., sediments and openwater environmental samples) both Maes et al. (2017) and 


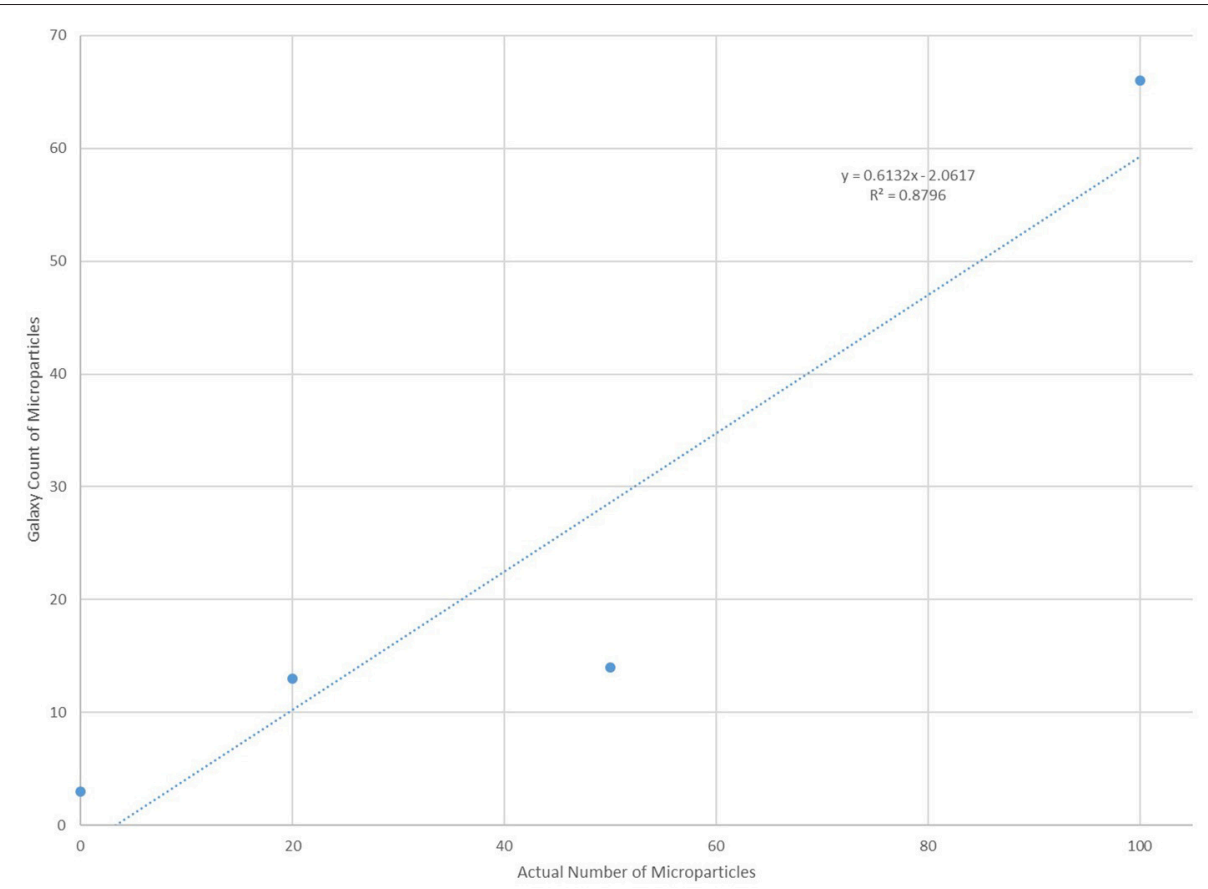

FIGURE 4 | Comparison of counts using the "Galaxy Count" software relative to the known number of microplastic particles within four test solutions.

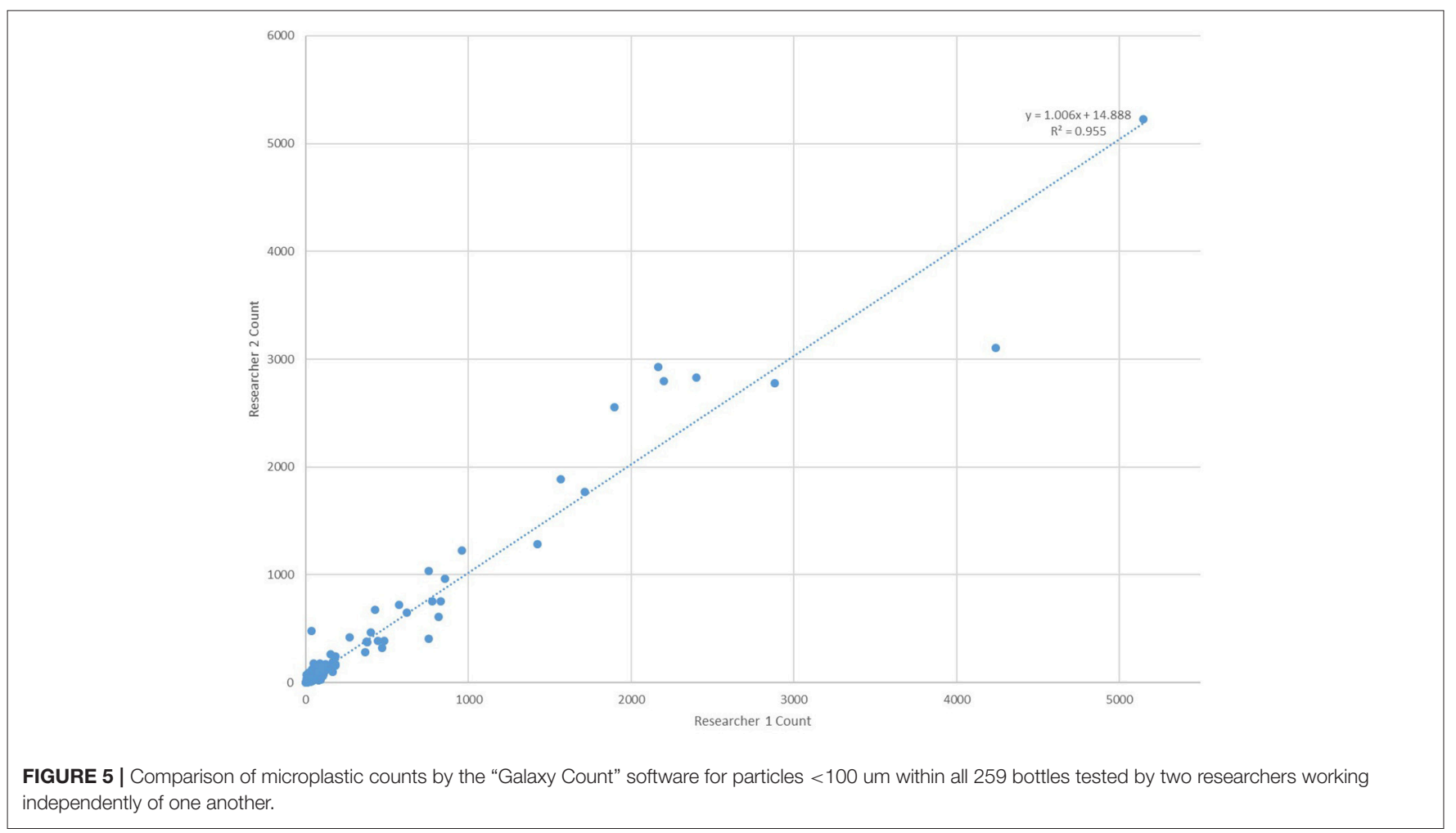

Erni-Cassola et al. (2017) concluded that Nile Red (NR) was very selective, especially within the time scales of incubation employed, and could be used for the rapid detection of microplastics without the need for additional spectroscopic analysis. To be sure that is why this stain was employed for this study. Additionally FTIR analysis was done on fluorescing particles $>100$ um and every particle analyzed was confirmed to be polymeric. Even further, NR is well-established to selectively 
adsorb to hydrophobic ("water-fearing") materials and, as such, will not adsorb to the only contents reasonably expected to be within bottled water, water and/or its mineral components. In addition, Schymanski et al. (2018) reported Raman confirmed densities of particles within a similar size range and even smaller (5-500 um) in bottles of German bottled mineral water. Thus, at a minimum while particles $<100$ um were not spectroscopically confirmed to be microplastics, particles are rationally expected to be plastic or of some other anthropogenic origin.

\section{DISCUSSION}

Part of the impetus for this study was as a follow-up to a tap water study released (in part) in September 2017 (Kosuth et al., 2018). The methods used in this study differed slightly in comparison to this earlier study, most notably in the use of a different stain. Rose Bengal was used in the earlier study, while Nile Red was used here. The two dyes have opposite affinities. While plastics adsorb Nile Red (allowing their easy detection via fluorescence), they do not adsorb Rose Bengal. The affinity of plastics to adsorb Nile Red allows smaller particles to be detected as compared to the Rose Bengal method, as noted by a recent study by Erni-Cassola et al. (2017). Thus, only our data on particles $>100$ um is comparable to the data in this previous tap water study.

We found roughly twice as many plastic particles (>100 um) within bottled water as compared to tap water on average (10.4 vs. 5.45 particles/L). While fibers made of $97 \%$ of the microplastics within the tap water study, they only composed $13 \%$ of the particles within bottled water. Instead fragments were the most common particle morphology (65\%) within bottled water. These results indicate that the main source of the microplastic particulate is different. Given the fragment morphology combined with the fact that $4 \%$ of the particles were found to have signatures of industrial lubricants, the data seems to suggest that at least some of the plastic contamination may be coming from the industrial process of bottling the water itself. As polypropylene was the most common polymer found, the fragments could also be breaking off the cap, even entering the water through the simple act of opening the bottle.

More recently Schymanski et al. (2018) published their study on microplastic contamination of packaged mineral water. They tested a wider variety of packaging media from returnable and single-use plastic bottles to cartons to glass, while this study almost exclusively focused on single-use plastic bottles (having only one lot packaged in glass as an alternative). They did test fewer bottles overall as compared to this study. In order to compare these two studies, then, only their data for single-use, plastic beverage bottles is utilized. Within those confines, they tested a total of 11 bottles in comparison to our 259. While they do not specify how many different brands, for one brand they tested two different lots (purchased 6 weeks apart), but only tested one lot for the others.

The average microplastic density across all brands, lot numbers and bottles analyzed (325 MPP/L) is significantly higher in this study as compared to that reported by Schymanski et al. (2018) (14 MPP/L). This difference could be owing to a number of factors. First, as they report they only counted particles for which they could fully confirm the polymeric nature using Raman spectroscopy. We used the adsorption of Nile Red as our frontline confirmation of microplastic identity, using FTIR on particles simply to provide more information as to the specific polymer. As the authors note, while Raman can analyze smaller particles than FTIR, the laser intensity can cause the particle to decompose before an adequate spectra can be obtained. Schymanski et al. (2018) did not include these particles in their counts leading to a reduction in their calculated densities. Further, as our data shows there can be substantial variability between brands and between lots. Our significantly larger sample set provides a greater accounting of that variability.

Another difference between our studies is distribution of polymer types. Schymanski et al. (2018) found PEST (the combination of polyester and polyethylene terephthalate) to be the dominant polymeric material of their particulate contaminants, while that same categorization only accounted for $6 \%$ of our analyzed particles. Here polypropylene was found to be the dominant plastic (54\%), which only accounted for $1 \%$ of their particles. However, our two studies are not fully comparable with regard to this analysis. Schymanski et al. (2018) analyzed and determined polymeric identity for all particles counted, while we only did so for particles $>100 \mathrm{um}$. It is quite possible that the smaller particles we were unable to analyze were mainly composed of the polymers within the PEST category, which would very much alter our percentages. Nevertheless, we both do reason from our data that the packaging of the water itself is a likely source of contamination, though for us it appears to be the caps, while for Schymanski et al. (2018) it appeared to be the bottle.

Despite the differences between our studies some similarities do exist. We both found polyethylene accounting for $\sim 10 \%$ of the polymeric contaminants. Additionally, we both found smaller particles provided a larger contribution to the total number of particles as compared to the larger particles (>100 um). Across all samples, $95 \%$ of our particles were $<100$ um, while Schymanski et al. (2018) found they accounted for $98 \%$ of their counts. Even further, taken together, these two studies do support the very basic point that there are microplastics within bottled water and at least some of this contamination may arise from the industrial process of bottling the water, as well as from the packing material itself.

\section{CONCLUSIONS}

Twenty-seven different lots of bottled water from 11 different brands purchased in 19 locations across nine different countries were analyzed for microplastic contamination using a Nile Red stain, which adsorbs to polymeric material and fluoresces under specific wavelengths of incident light. The use of the fluorescent dye allowed for smaller particles to be detected as compared to a similar study of tap water using a Rose Bengal stain, though the analytical methods employed for their enumeration restricted the lower size limit to $6.5 \mathrm{~mm}$. 
Of the 259 total bottles analyzed, 93\% showed signs of microplastics. There was significant variation even among bottles of the same brand and lot, which is consistent with environmental sampling and likely resulting from the complexities of microplastic sources, the manufacturing process and particle-fluid dynamics, among others. As bottle volume varied across brands, absolute particle counts were divided by bottle volume in order to produce microplastic particle densities that were comparable across all brands, lots and bottles. These densities were reduced by lab blanks in order to account for any possible contamination. Given our use of lab blanks, the inability to photograph the full filter, the lower limit of one pixel being equivalent to $6.5 \mathrm{~mm}$, and control runs of the software employed to digitally count particles $<100 \mathrm{~mm}$, the numbers reported here are very conservative and likely undercounting, especially with regard to smaller microplastics $(<100 \mathrm{~mm})$, which were found to be more prominent (on average $95 \%$ ) as compared to particles $>100 \mathrm{~mm}$ (on average 5\%).

Infrared analysis of particles $>100 \mathrm{~mm}$ in size confirmed microplastic identity and found polypropylene to be the most common (54\%) polymeric material (at least with regard to these larger microplastics), consistent with a common plastic employed to manufacture bottle caps. Smaller particles $(6.5-100 \mathrm{~mm})$ could not be analyzed for polymer identification given the analytical limits of the lab. While these smaller particles could not be spectroscopically confirmed as plastic, Nile Red adsorbs to hydrophobic ("water-fearing") materials, which are not reasonably expected to be naturally found within bottled water. Our FTIR analysis of larger (>100 um particles) fluorescing particles, all of which were confirmed to be polymeric, provides additional support of the selective binding of NR to microplastic particles within the samples. Even further, Schymanski et al. (2018) did spectroscopically confirm (via Raman) particles within this smaller size range in German bottled water as being polymeric in nature provide additional support for their presence. Given this and following the conclusions of prior studies (Erni-Cassola et al., 2017; e.g., Maes et al., 2017) the adsorption of Nile Red alone was used to confer microplastic identity to these smaller particles. As the specific polymer content could not be determined, they could very well show a different compositional pattern as compared to the larger particles analyzed. This could explain the difference in our polymeric compositional analysis relative to a very recent and similar analysis of bottled mineral waters by Schymanski et al. (2018), which found PEST (polyester+polyethylene terephthalate) to

\section{REFERENCES}

Baldwin, A. K., Corsi, S. R., and Mason, S. A. (2016). Plastic debris in 29 great lakes tributaries: relations to watershed attributes and hydrology. Environ. Sci. Technol. 50, 10377-10385. doi: 10.1021/acs.est.6b 02917

Brennecke, D., Ferreira, E. C., Costa, T. M., Appel, D., da Gama, B. A., and Lenz, M. (2015). Ingested microplastics are translocated to organs of the tropical fiddler crab Uca rapax. Mar. Pollut. Bull. 96, 491-495. doi: 10.1016/j.marpolbul.2015.05.001 be the most common polymeric material, consistent with a common plastic employed to manufacture the bottle itself. Either way both studies indicate that at least part of the microplastic contamination is arising from the packaging material and/or the bottling process itself.

Beyond the polymeric identity of the microplastics, the morphology of the particles also provides an indication as to a different source of contamination relative to an earlier study on globally sourced tap water. In this prior study $83 \%$ of the 159 samples were show to contain anthropogenic debris and 98\% of those particles were microfibers. In comparison, this study found microplastic contamination within $93 \%$ of the individual bottles (and in all of the brands and lots tested) with only $13 \%$ of the particles being categorized as microfibers. The vast majority (65\%) of the microplastics were identified as fragments indicating a different source of the contamination relative to the tap water. Even further, the bottled water contained on average nearly twice as much microplastic contamination (within the same size range, i.e., $>100 \mathrm{um}$ ) as compared to tap water (10.4 vs. 5.45 particles/L). While the impacts of microplastic contamination on human health are still unknown, these results strongly support a reduction in the bottling of water and in the consumption of bottled water, especially within locations in which clean, safe tap water exists.

\section{DATA AVAILABILITY}

The raw data supporting the conclusions of this manuscript will be made available by the authors, without undue reservation, to any qualified researcher.

\section{AUTHOR CONTRIBUTIONS}

SM designed the study, supervised the work, ensured quality control and wrote the manuscript. VW was the lead laboratory research assistant and conducted all aspects of the laboratory analysis. JN assisted in and conducted laboratory analyses.

\section{ACKNOWLEDGMENTS}

The authors wish to thank Orb Media who conducted the market analysis to determine the top selling bottled water brands within each region and facilitated the purchase and delivery of all samples to our lab. 
Erni-Cassola, G., Gibson, M. I., Thompson, R. C., and Christie-Oleza, J. (2017). Lost, but found with Nile Red: a novel method to detect ad quantify small microplastics (20 um-1 mm) in environmental samples. Environ. Sci. Technol. 51, 13641-13648. doi: 10.1021/acs.est.7b04512

Hidalgo-Ruz, V., Gutow, L., Thompson, R. C., and Thiel, M. (2012). Microplastics in the marine environment: a review of the methods used for identification and quantification. Environ. Sci. Technol. 46, 3060-3075. doi: 10.1021/es2031505

Horton, A. A., Walton, A., Spurgeon, D. J., Lahive, E., and Svendsen, C. (2017). Microplastics in freshwater and terrestrial environments: Evaluating the current understanding to identify the knowledge gaps and future research priorities. Sci. Total Environ. 586, 127-141. doi: 10.1016/j.scitotenv.2017.01.190

Iñiguez, M. E., Conesa, J. A., and Fullana, A. (2017). Microplastics in Spanish Table Salt. Sci. Rep. 7, 8620-8627. doi: 10.1038/s41598-017-09128-x

Karami, A., Golieskardi, A., Choo, C. K., Larat, V. T., Galloway, T., and Salamatinia, B. (2017). The presence of microplastics in commercial salts from different countries. Sci. Rep. 7, 46173-46184. doi: 10.1038/srep46173

Kosuth, M., Mason, S. A., and Wattenberg, E. V. (2018). Anthropogenic contamination of tap water, beer, and sea salt. PLOS ONE 13:e0194970. doi: 10.1371/journal.pone.0194970

Liebezeit, G., and Liebezeit, E. (2014). Synthetic particles as contaminants in German beers. Food Addit. Contam. 31, 1574-1578. doi: 10.1080/ 19440049.2014.945099

Lusher, A. L., Hollman, P. C. H., and Mendoza-Hill, J. J. (2017). Microplastics in Fisheries and Aquaculture: Status of Knowledge on Their Occurrence and Implications for Aquatic Organisms and Food Safety. FAO Fisheries and Aquaculture Technical Paper. No. 615. Royal Society of Chemistry, Rome.

Maes, T., Jessop, R., Wellner, N., Haupt, K., and Mayes, A. G. (2017). A rapid-screening approach to detect and quantify microplastics based on fluorescent tagging with Nile Red. Sci. Rep. 7, 44501-44511. doi: 10.1038/srep 44501

Moore, C. J., Moore, S. L., Leecaster, M. K., and Weisberg, S. B. (2001). A comparison of plastic and plankton in the North Pacific Central Gyre. Mar. Pollut. Bull. 42, 1297-1300. doi: 10.1016/S0025-326X(01) 00114-X

PlasticsEurope (2015). Plastics - The Facts 2015: An Analysis of European Plastics Production, Demand and Waste Data. Belgium: PlasticsEurope.

Renner, G., Schmidt, T. C., and Schram, J. (2018). Analytical methodologies for monitoring micro(nano)plastics: which are fit for purpose? Environ. Sci. Health, 1, 55-61. doi: 10.1016/j.coesh.2017.11.001
Rochman, C. M., Tahir, A., Williams, S. L., Baxa, D. V., Lam, R., Miller, J. T., et al (2015). Anthropogenic debris in seafood: plastic debris and fibers from textiles in fish and bivalves sold for human consumption. Sci. Rep. 5, 14340-14350. doi: $10.1038 /$ srep 14340

Schymanski, D., Goldbeck, C., Humpf, H. U., and Fürst, P. (2018). Analysis of microplastics by micro-Raman spectroscopy: release of plastic particles from different packaging into mineral water. Water Res. 129, 154-162. doi: 10.1016/j.watres.2017.11.011

Sharma, S., and Chatterjee, S. (2017). Microplastic pollution, a threat to marine ecosystem and human health: a short review. Environ. Sci. Pollut. Res. 24, 21530-21547. doi: 10.1007/s11356-017-9910-8

Tanaka, K., and Takada, H. (2016). Microplastic fragments and microbeads in digestive tracts of planktivorous fish from urban coastal waters. Sci. Rep. 6, 34351-34359. doi: 10.1038/srep34351

UNEP (2016). Marine Plastic Debris and Microplastics: Global Lessons and Research to Inspire Action and Guide Policy Change. Nairobi: United Nations Environment Programme.

Van Cauwenberghe, L., and Janssen, C. R. (2014). Microplastics in bivalves cultured for human consumption. Environ. Pollut. 193, 65-70. doi: 10.1016/j.envpol.2014.06.010

Yang, D., Shi, H., Li, L., Jabeen, K., and Kolandhasamy, P. (2015). Microplastic pollution in Table Salt from China. Environ. Sci. Technol. 49, 13622-13627. doi: 10.1021/acs.est.5b03163

Yonkos, L. T., Friedel, E. A., Perez-Reyes, A. C., Ghosal, S., and Arthur, C. A. (2014). Microplastics in four estuarine rivers in the Chesapeake Bay, USA. Environ. Sci. Technol. 48, 14195-14202. doi: 10.1021/es50 36317

Conflict of Interest Statement: The authors declare that the research was conducted in the absence of any commercial or financial relationships that could be construed as a potential conflict of interest.

Copyright (C) 2018 Mason, Welch and Neratko. This is an open-access article distributed under the terms of the Creative Commons Attribution License (CC BY). The use, distribution or reproduction in other forums is permitted, provided the original author(s) and the copyright owner(s) are credited and that the original publication in this journal is cited, in accordance with accepted academic practice. No use, distribution or reproduction is permitted which does not comply with these terms. 